\title{
Influencia de las fuentes de financiamiento externo en el crecimiento y desarrollo de las empresas agroindustriales del distrito de Abancay Apurímac Perú (periodo 2012- 2013)
}

\author{
Wilber Jiménez Mendoza ${ }^{1}$ \\ Rober Anibal Luciano Alipio ${ }^{2}$ \\ Carolina Soto Carrión ${ }^{3}$
}

\section{Resumen}

En los últimos años, el Perú viene creciendo sostenidamente, la tasa de crecimiento del PBI ha superado el 7\%, lo que nos indica que estamos atravesando por una bonanza económica; sin embargo esta se explica por la explotación y exportación básicamente de materias primas como minerales, la construcción y los productos agroindustriales.

Apurímac no es ajeno a esta realidad, si bien es cierto, contribuye aproximadamente con el $0.5 \%$ del PBI nacional, se prevé un futuro más halagador, principalmente por el potencial minero que tiene. A la vez sabemos que este sector es efímero.

En la presente investigación se abordó el estudio del crecimiento y desarrollo de las empresas agroindustriales del distrito de Abancay, influenciado por las fuentes de financiamiento externo.

Palabras clave: fuentes de financiamiento externo, crecimiento de las empresas, desarrollo de la empresa.

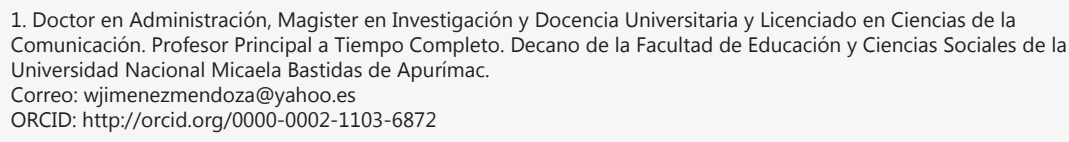

2. Magister en Administración y Licenciado en Administración, Profesor Asociado a tiempo completo de la Facultad de Administración de la Universidad Nacional Micaela Bastidas de Apurímac.

ORCID: http://orcid.org/0000-0001-9555-6690

3. Doctora en Medio Ambiente y Desarrollo Sostenible, Magister en Investigación y Docencia Universitaria. Profesora Principal a Tiempo Completo de la Universidad Tecnológica de los Andes de Apurímac. Directora de la Oficina de Relaciones Internacionales. 


\title{
Influence of external funding sources in the growth and development of agrarian business District Abancay Apurimac Peru (period 2012-2013)
}

\begin{abstract}
In recent years, Peru has been growing steadily, the rate of GDP growth exceeded $7 \%$, indicating that we are going through an economic boom; but this is explained by the exploitation and export of raw basically one hand as minerals, construction and agro industrial products on the other.

Apurimac is no stranger to this reality, although, contributes about $0.5 \%$ of national GDP, is forecast to more flattering future, mainly because of the potential for mining. While we know that this area is ephemeral.

In this research study the growth and development of agrarian business in the Abancay district, influenced by sources of external financing.
\end{abstract}

Keywords: Sources of External Financing. Growth Companies. Development Company.

Recibido: 05-03-2014

Aceptado: 05-05-2014

\section{lntroducción}

Hablar de las empresas agroindustriales del distrito de Abancay, es hablar de empresas que en su mayoría se encuentran en lento crecimiento y pocas son las que han alcanzado su madurez. En el distrito se cuenta con 212 empresas agroindustriales, con RUC activo ante la Superintendencia Nacional de Aduanas y de Administración Tributaria (SUNAT).

Dichas empresas a la fecha se catalogan como micro empresas, en su mayoría, ya que sus activos y sus ventas son mínimos. Por otro lado se puede evidenciar que, a la fecha se gestionan en su mayoría en forma empírica, manejadas principalmente por emprendedores que buscan aprovechar las oportunidades del valle de Abancay, y de la presencia de mercados, para producir productos que a la fecha tienen alta demanda. 
Las empresas agroindustriales, se dedican principalmente a la transformación de productos agrícolas, y en menor medida a la transformación de productos pecuarios. Los factores limitantes de mayor trascendencia, para el crecimiento y desarrollo de las empresas agroindustriales, son las fuentes de financiamiento, la capacitación en procesos productivos, dificultades en gestión empresarial y en acceso a mercados.

El presente estudio abordó el primer aspecto de la problemática planteada en el párrafo anterior, es decir; las fuentes de financiamiento externo y su influencia en el crecimiento y desarrollo de las empresas agroindustriales. Terminando con la presentación de las conclusiones y recomendación de la presente investigación.

\section{Material y métodos}

\section{Tipo de investigación}

La investigación desarrollada es de tipo básica, porque no tiene propósitos aplicativos inmediatos, solo busca ampliar y profundizar el caudal de conocimientos científicos (Charaja, 2009: 41).

\section{Nivel de investigación}

El nivel de investigación es explicativa y causal ya que está dirigida a responder a las causas de los eventos físicos o sociales (...) su interés se centra en conocer por qué ocurre un fenómeno (...) más estructurados y profundizados que los demás niveles de estudio (Charaja, 2009: 44).

\section{Métodos de investigación}

Para la investigación se aplicó en primer lugar el método de la observación del objeto de investigación, luego se usó el método del análisis para identificar las variables y finalmente el método deductivo, de la teoría general al objeto particular de investigación.

El diseño de investigación corresponde al método científico de las ciencias sociales con un carácter no experimental y la recopilación de los datos en forma transversal, utilizando los tipos de hipótesis nula y alternativa con una medición estadística.

\section{Resultados}

Las empresas agroindustriales analizadas en la presente investigación, fueron realizadas, con las técnicas de observación y encuesta; se pudo evidenciar que el sector en el distrito de Abancay se encuentra en la fase de crecimiento en su mayoría, debido a que el sector no se encuentra muy desarrollado. 
Así mismo se observa que las inversiones realizadas en las empresas en su mayoría son escasas, constituidas principalmente por empresas familiares, que brindan puestos de trabajo a los integrantes de la familia.

De la encuesta realizada se pudieron obtener los siguientes datos:

De los 137 encuestados, el 49\% de los mismos son personas que se ubican en el rango de 40 a 60 años, lo que demuestra que son personas adultas con negocios consolidados, mientras que el $27 \%$ se encuentra en el grupo de 20 a 40 años de edad, se deduce que son personas jóvenes emprendedoras y que tienen negocios generalmente en inicio o en fase de crecimiento; por otro lado se puede evidenciar que un $19 \%$ de encuestados tiene entre 60 a 80 años, como adultos mayores que son, cuentan con negocios familiares y 5\% de adultos mayores son de más de 80 años y representa a personas que generalmente se encuentran a cargo de negocios tradicionales, finalmente se registra un $0 \%$ de menores de 20 años.

Por otro lado, de los encuestados, el $82 \%$ corresponde al sexo masculino y el restante $18 \%$ al sexo femenino.

Del análisis realizado a las empresas, respecto al valor de los activos propios, el $91 \%$ tiene menos de S/. 100,000.00 nuevos soles, mientras que tan solo el $9 \%$ de sus activos valora más de S/. 100,000.00 nuevos soles, como valor de activos ${ }^{4}$.

De las empresas analizadas se encontró como resultado, que el $90 \%$ de las empresas tiene menos de diez trabajadores, mientras que un $10 \%$ cuenta con más de diez y menos de veinte trabajadores. Lo que demuestra que las empresas son pequeñas y se encuentran en crecimiento.

La mayoría de empresas, $88 \%$ cuenta con un solo local, principalmente de producción, mientras que el $8 \%$ manifiesta que cuentan con dos locales y el 4\% con tres locales que son de producción y de comercialización en la mayoría de los casos.

De la información obtenida por la encuesta, también se puede deducir que el $64 \%$ de las empresas se dedica a la producción de menos de tres productos, mientras que el $22 \%$ produce entre 3 a 6 productos; el $7 \%$ produce entre 7 a 9 productos y el $7 \%$ restante manifiesta que producen de 10 a más productos agroindustriales.

\section{Fuentes de financiamiento}

Del análisis realizado a los datos obtenidos, respecto a las fuentes de financiamiento externo, es decir recursos financieros provenientes de personas naturales o jurídicas ajenas a la empresa; se ha obtenido como resultado que para 
el año 2012 el 80\% de las empresas han utilizado este tipo de recursos, mientras que el restante 20\% no lo hizo. Por otro lado para el año 2013 el $81 \%$ hizo uso de este tipo de recursos y el 19\% no utilizó este tipo de fuente.

Lo que demuestra que en los periodos en estudio, las empresas en su gran mayoría hicieron uso de fuentes externas de financiamiento.

Si realizamos un análisis, al número de fuentes de financiamiento utilizadas por las empresas agroindustriales, prevalece el uso de dos fuentes de financiamiento en el año 2012; es así que el 54\% había utilizado dos fuentes externas, entre bancos y préstamos familiares, seguido de tres fuentes de financiamiento en un $23 \%$ de los casos, mientras que el 12\% utilizó más de tres fuentes de financiamiento y el $11 \%$ tan solo una fuente de financiamiento externo.

En el año 2013, 47\% de los casos utilizó dos fuentes de financiamiento, 26\% tres fuentes de financiamiento, $17 \%$ más de tres fuentes y el 10\% utilizó una fuente de financiamiento.

Si analizamos el monto del financiamiento al que se recurrió, según los datos obtenidos, el $41 \%$ de las empresas agroindustriales solicitó un fondo entre S/. $20,001.00 \mathrm{a} \mathrm{S} / .30,000.00$ nuevos soles en el año 2012 y $42 \%$ en este mismo monto en el año 2013; entre S/ 10,001.00 a S/. 20,000.00 nuevos soles, el 39\% recurrió en el año 2012, y 30\% el mismo monto también en el año 2013.

Mientras un $13 \%$ hizo uso en los años 2012 y $21 \%$ en el año 2013 por un monto entre S/ 30,001.00 a S/. 40,000.00 nuevos soles, como los más importantes; finalmente los que obtuvieron como montos mayores a S/. 40,000.00 fue del 5\% el año 2012 y 4\% en el año 2013 y 2\% menos de S/10,000.00 en el año 2012 y $4 \%$ el mismo monto en el año 2013.

$\mathrm{Si}$ realizamos un análisis del monto al que accedieron las empresas agroindustriales, de las fuentes de financiamiento, se ha determinado que para el año 2012 el monto promedio fue de S/ 23,157.89 nuevos soles, mientras que para el año 2013 este monto fue de S/. 24,122.81 nuevos soles, lo que indica que fue superior al año anterior.

El costo al cual accedieron las empresas agroindustriales respecto de las fuentes de financiamiento, es decir la tasa de interés para el año 2012, fue que el $35 \%$ contrato a una tasa de interés, de entre $2 \%$ y $2.5 \%$ de interés mensual; el $44 \%$ de las empresas accedió entre el 1.5\% y 2\% de interés mensual; el 3\% a una tasa mayor $2.5 \%$ de interés mensual; $15 \%$ entre $1 \%$ y $1.5 \%$ mensual y $4 \%$ menos del $1 \%$ de tasa de interés.

Para el año 2013, los costos de las fuentes de financiamiento fueron alrededor de un $39 \%$ de los casos entre $2 \%$ y $2.5 \%$ de interés mensual; el $44 \%$ asumió un costo entre $1.5 \%$ a $2 \%$ de interés mensual; $8 \%$ mayor a $1 \%$ mensual; finalmente $13 \%$ entre $1 \%$ y $1.5 \%$ mensual y $3 \%$ menos de $1 \%$ mensual. 
El periodo de tiempo de las fuentes de financiamiento, a las cuales se ha contratado los fondos, en el año 2012 se tiene un $25 \%$ en un periodo entre $1 \mathrm{y}$ 2 años; así como el $58 \%$ entre 2 y 3 años; $11 \%$ entre 3 y 4 años y $2 \%$ manifiesta que el periodo al cual accedió la fuente de financiamiento fue mayor a 4 años.

Para el año 2013, sin embargo el 41\% de los casos accedió a un periodo entre 2 a 3 años; $26 \%$ un plazo entre 1 y 2 años, $12 \%$ en un periodo entre 3 y 4 años y $4 \%$ mayor a 4 años.

Por otro lado, también se ha analizado si para hacer uso de las fuentes de financiamiento externo, fue necesario hacer uso de garantía en el año 2012, lo que muestra que el $84 \%$ sí lo hizo; mientras que para el $16 \%$ no fue necesario. Así mismo en el año 2013, el $82 \%$ hizo uso de garantías y para el $18 \%$ de las empresas no fue requisito.

\section{Crecimiento empresarial}

En relación al crecimiento empresarial, los empresarios de las organizaciones agroindustriales del distrito de Abancay, consideran que los recursos obtenidos por las fuentes de financiamiento externo, al aplicarlos en su empresa han tenido impactos que se determinaron de la siguiente forma: se observa que para el año 2012, el 45\% considera que las fuentes de financiamiento externo ha tenido un impacto medio en el crecimiento de la empresa, mientras que el $41 \%$ piensa que el impacto fue bajo y el $1 \%$ cree que el impacto fue alto.

Para el año 2013, el 46\% considera que las fuentes de financiamiento externo han impactado en un nivel medio, mientras que el $44 \%$ piensa que el crecimiento fue bajo y $1 \%$ considera que fue alto.

Analizando los puestos de trabajo generados por las empresas agroindustriales, en lo referente a recursos humanos, se observa que en el año 2012 se crearon tres puestos de trabajo en el $13 \%$ de las empresas, dos puestos de trabajo en el $34 \%$, un puesto en el $24 \%$, y ninguno en el $29 \%$.

En el año 2013, se puede notar que las empresas crearon tres puestos de trabajo en el $13 \%$ de ellas, dos puestos en un $34 \%$, un puesto en el $24 \%$ y ningún puesto de trabajo en el $28 \%$.

En las empresas agroindustriales del distrito de Abancay, en el año 2012, el 50\% ha adquirido un activo fijo tangible, 28\% dos activos, $9 \%$ tres activos, $1 \%$ más de tres activos y $12 \%$ ningún activo.

Mientras que en el año 2013, se observa que el 45\% ha adquirido un activo fijo tangible, $32 \%$ dos activos, $10 \%$ tres activos, $2 \%$ más de tres activos y $11 \%$ ningún activo. 
Las empresas agroindustriales, en lo que se refiere a recursos financieros, en el año 2012, el 66\% de los casos manejó un recurso financiero, el 20\% manejó dos recursos financieros, el $6 \%$ tres recursos, el 1\% más de tres y el $7 \%$ ningún recurso financiero.

En el año 2013, el 70\% de los casos manejó un recurso financiero, el 17\% manejó dos recursos financieros, el 4\% tres recursos, el 1\% más de tres y el $8 \%$ ningún recurso financiero. Como se muestra en el siguiente gráfico:

Al ser consultados los empresarios agroindustriales, respecto a los recursos financieros, estos manifiestan que en el periodo 2012, el $45 \%$ manifiesta que disminuyó poco, el $45 \%$ que no ha variado, el $7 \%$ que se incrementó poco, el $0 \%$ que incrementó bastante, y el 4\% que disminuyó bastante.

Mientras que para el periodo 2013, manifiestan en un 50\% que disminuyó poco, $44 \%$ que no ha variado, $1 \%$ que se incrementó poco, $1 \%$ se incrementó bastante y $4 \%$ que disminuyó bastante.

Analizando la tecnología de producción de las empresas agroindustriales, en el periodo 2012, se observa que el $0 \%$ realizaba los productos en forma industrial, $61 \%$ en forma semi industrial, $36 \%$ en forma artesanal y $2 \%$ sin ningún valor agregado, es decir solo se dedica a la comercialización.

En el periodo 2013, el 0\% utiliza una tecnología de producción industrial, el 63\% semi industrial, el $36 \%$ de forma artesanal y un $1 \%$ sin ningún valor agregado.

\section{Desarrollo empresarial}

Al analizar las apreciaciones respecto al desarrollo de las empresas agro industriales del distrito de Abancay, y si este desarrollo fue motivado por las fuentes de financiamiento, los resultados fueron para el año 2012, que el 15\% considera que el impacto fue medio, el 30\% bajo, el 36\% ningún impacto y $3 \%$ que el impacto fue alto.

En el año 2013, el 24\% considera que el impacto fue medio, 34\% considera un impacto bajo, $26 \%$ ningún impacto y $0 \%$ un impacto alto.

Respecto a la participación de mercado de las empresas agroindustriales del distrito de Abancay, en el periodo 2012, el 27\% considera tener una participación media, 4\% una participación alta, $46 \%$ una participación baja, 24\% una participación muy baja y $0 \%$ una participación muy alta.

En el periodo 2013, el 32\% considera que tiene una participación de mercado media, $48 \%$ de participación baja, $5 \%$ una participación alta y $15 \%$ considera una participación muy baja. 
Respecto al número de mercados que atiende las empresas agroindustriales del distrito de Abancay, en el periodo 2012, el 12\% atiende a un solo mercado, que en su mayoría es un mercado local; el 59\% atiende dos mercados entre locales y regionales, el $28 \%$ tres mercados, el $0 \%$ cuatro mercados y el $0 \%$ más de cinco mercados.

En el periodo 2013, el 15\% de las empresas atiende en un solo mercado, el 58\% atiende a dos mercados, el $26 \%$ a tres mercados, el $1 \%$ cuatro mercados, y el $0 \%$ más de cinco mercados.

En las empresas agroindustriales se observan que para el año 2012, el 86\% ha desarrollado productos nuevos, de las cuales el 55\% ha desarrollado tan solo un producto, el 30\% desarrolló dos productos nuevos, y el 1\% tres productos nuevos; mientras que el $15 \%$ de las empresas no desarrolló ningún producto.

En el periodo 2013, el 86\% de las empresas desarrolló nuevos productos, de los cuales el 53\% desarrolló un producto nuevo, el 31\% dos productos nuevos y el $2 \%$ tres.

Respecto al desarrollo en gestión empresarial, las empresas manifiestan que en el periodo 2012, el 42\% contaba con un documento de gestión y/o proceso, el $37 \%$ con dos, el $1 \%$ con tres documentos y el $1 \%$ con más de tres documentos; mientras que el 18\% manifiesta que no cuenta con documentos de gestión y/o procesos administrativos. En el periodo 2013, el 39\% contaba con un documento de gestión y/o proceso, el $41 \%$ con dos, el 2\% con tres documentos y el $1 \%$ con más de tres documentos; mientras que el $17 \%$ manifiesta que no contaba con documentos de gestión y/o procesos administrativos, en el referido periodo.

Realizando un análisis de las unidades orgánicas de las empresas agroindustriales del distrito de Abancay, en el periodo 2012, el 64\% contaba con dos áreas, el $31 \%$ con tres áreas y el $4 \%$ con una sola área.

En el periodo 2013, el 64\% contaba con dos áreas, el 33\% con tres áreas y el 4\% con una sola área.

Por otro lado, realizando un análisis del ciclo de vida de las empresas, en el periodo 2012, el 5\% considera que se encuentra en una fase de inicio, $69 \%$ en la fase de crecimiento, $26 \%$ en fase de madurez y $1 \%$ en fase de decrecimiento.

Para el periodo del 2013, el 5\% considera que se encuentra en una fase de inicio, $69 \%$ en la fase de crecimiento, $26 \%$ en una fase de madurez y $1 \%$ en una fase de decrecimiento. 


\section{Discusión}

Se ha demostrado empíricamente que las fuentes de financiamiento externo son muy importantes para el crecimiento y desarrollo de las empresas agroindustriales del distrito de Abancay, las mismas que se demuestra en el alto uso de las fuentes de financiamiento por las empresas.

Sin embargo se debe de tener en cuenta los diferentes factores al momento de acceder a ellas, como son el plazo, la tasa de interés y el monto.

Durante el tiempo de trabajo de campo, efectuado en los meses de marzo a mayo del último periodo de investigación, no se ha observado la relación estrecha que existe entre los empresarios y los ofertantes de financiamiento, principalmente de los bancos, cajas municipales, cajas rurales, cooperativas de ahorro y crédito y EPYMEs.

Asimismo, se ha visto una fuerte relación con los proveedores de materiales, insumos y servicios, quienes brindan créditos que sirven como fuente de financiamiento.

Por otro lado, se pudo evidenciar la participación familiar en el financiamiento, principalmente de las empresas que están en fase de inicio.

Del mismo modo es de resaltar que las empresas agroindustriales, en su mayoría, se encuentran en fase de crecimiento y un mínimo porcentaje de ellas se encuentran en la fase de madurez.

Por lo que se pudo notar que las empresas están creciendo en recursos físicos, humanos y financieros. Ya que vienen adquiriendo activos fijos, creando nuevos puestos de trabajo y bancarizando su economía.

Finalmente en cuanto al desarrollo es más lento, en lo que se refiere a participación en el mercado, diversificación de nuevos productos, penetración en nuevos mercados y manejo de gestión empresarial.

\section{Conclusiones}

La investigación realizada nos permite llegar a los siguientes resultados y conclusiones: 
Hemos demostrado que durante la contrastación de la hipótesis principal, con el Valor $\mathrm{p}=0.000<0.05$, que las fuentes de financiamiento influyen positivamente en un alto nivel de significancia con el crecimiento y desarrollo de las empresas agroindustriales del distrito de Abancay en los periodos 2012 y 2013, con una correlación positiva de Nivel Alto de $79.40 \%$.

En el análisis de la variable fuentes de financiamiento y crecimiento empresarial, cómo el Valor $\mathrm{p}=0.000<0.05$, podemos afirmar que las fuentes de financiamiento externo se relacionan significativamente con el crecimiento empresarial de las empresas agroindustriales del distrito de Abancay, con una correlación positiva de nivel medio de $68.9 \%$.

Del mismo modo en el análisis de la variable fuentes de financiamiento externo y desarrollo empresarial, como el Valor $\mathrm{p}=0.000<0.05$ podemos afirmar que existe una relación significativa entre las fuentes de financiamiento y desarrollo empresarial de las empresas agroindustriales del distrito de Abancay en los periodos 2012 y 2013, con una correlación positiva de nivel medio de $67.6 \%$; asimismo, las fuentes de financiamiento más utilizadas por empresas investigadas, son las provenientes de los bancos, cajas municipales, cajas rurales, cooperativas de Ahorro y crédito y EPYMEs, así como créditos de proveedores y préstamos familiares como los más importantes.

Ha quedado demostrado que las fuentes de financiamiento influyen positivamente en el crecimiento y desarrollo empresarial, estas tiene que ver con los elementos de las fuentes como son el período de tiempo, las tasas de interés y los montos.

Por último, en las empresas en la ciudad de Abancay, se ha evidenciado que tienen un crecimiento significativo, ya que vienen creciendo en activos fijos, en nuevos empleos de trabajo y recursos financieros. Las empresas vienen desarrollando principalmente sus mercados y sus productos, sin embargo en menor medida se ve el desarrollo de los procesos de gestión en los que se ha evidenciado que tienen muchas falencias.

\section{Reconocimientos}

Para hacer uso de las fuentes de financiamiento externo se recomienda que éstas deban ser evaluadas en sus elementos componentes de tal manera que se pueda obtener financiamiento a mayor tiempo, con la menor tasa de interés posible y con el monto adecuado de acuerdo a sus necesidades. Con la finalidad de buscar un apalancamiento financiero adecuado que les permita maniobrar sus operaciones y obligaciones.

Asi mismo, las empresas para que puedan crecer, deben invertir más en activos fijos, como son compra de maquinaria, terrenos, equipo, muebles y enseres. De 
tal manera que les permita apalancarse operativamente a fin de reducir sus costos de producción y ser más competitivos.

Así mismo, se recomienda que en la creación de nuevos puestos de trabajo, éstos deban ser mayormente para personal capacitado y calificado a fin de garantizar la productividad de la empresa.

Es conveniente que las empresas se integren al sistema bancario, a fin de tener un aliado en las fuentes de financiamiento que les permita crecer de mejor forma.

Si las empresas logran un crecimiento, esto le permitirá el desarrollo de sus productos, de sus mercados y de la gestión empresarial. Ya que con mejores recursos, se podrán producir nuevos productos, enfrentar nuevos mercados y gestionar en forma más eficiente las empresas, que deben apuntar a una economía globalizada aprovechando los tratados de libre comercio.

\section{Referencias bibliográficas}

Aguiar, I. 1989. Rentabilidad y riesgo en el comportamiento financiero de la empresa. Las Palmas: Ed. CIES.

Albach, H. 1970. Un modelo de comportamiento del crecimiento de la empresa. Boletín de Estudios Económicos, vol. 9: 122-135.

Angenieux, G. 1969. Los ratios y la expansión de la empresa. Madrid: Ediciones ICE.

Blair, J. 1976. Economic Concentration, en CEA, J.L.: Comportamiento financiero de la empresa capitalista. Tesis Doctoral de la Universidad Autónoma de Madrid.

Brealey, R. \& Myers, S. 1993. Fundamentos de financiación empresarial. Madrid: Ed. McGraw-Hill.

Creehan, K. \& Bonomo, J.1996. De la intención a la acción: un proceso para identificar con toda precisión las oportunidades de crecimiento. Harvard Deusto Business Review, marzo- abril, $\mathrm{N}^{\circ}$ 71: 6-14.

Charaja, F. 2009. El MAPIC en la metodología de investigación. Puno: Ed. San Marcos.

Eco, U. 1991. Cómo se hace una tesis. Barcelona: Gedisa.

Enseñat de Villalonga, A. 1972. La tecnología como factor de dimensión. Economía Industrial, $\mathrm{N}^{\circ}$ 107: 132-133.

Friedman, M. 1966. Capitalismo y libertad. Madrid: Rialp. 
García, J. \& Martín, S. 1992. Métodos de desarrollo de las estrategias de crecimiento y diversificación empresarial. Actualidad Financiera, № 3: 61-74 (semana 20-26, enero).

González, J. 1988. Crecimiento y tamaño de la empresa. Una aplicación al sector textil catalán. Boletín de Información Comercial Española, noviembre, Vol. 43 (135): 503-520.

Hernández, M. 1997. El valor añadido como indicador económico de la responsabilidad social de la empresa: una aplicación empírica. Tesis Doctoral de la Universidad de La Laguna.

Hernández, R., Fernández, C. \& Baptista, P. 2010. Metodología de la Investigación. México: McGraw-Hill.

Kaldor, N. 1979. El equilibrio de la empresa. Lecturas de introducción a la economía de la empresa, Madrid: Pirámide.

Lizcano, J. 1987. La dimensión integral de la empresa: un enfoque contable. Madrid: Universidad Autónoma de Madrid.

Maurer, H. 1958. La gran empresa. Su desarrollo y funcionamiento. Buenos Aires: Edit. Selección Contable.

Myers, S. \& Majluf, N. 1984. Corporate Financing and Investment Decisions when Firms Have Information that Investors do not Have. Journal of Financial Economics, $\mathrm{N}^{\circ} 13$ (2): 187-221.

Peña, D. 1991. Estadística, modelos y métodos. Madrid: Alianza Universidad Textos.

Pérez, J. 1978. La dimensión óptima de la empresa. Madrid: Ibérico-Europea de ediciones S.A.

Raymond, J., Maroto, J. \& Melle, M. 1999. Inversión y crecimiento empresarial: factores condicionantes. Papeles de Economía Española, № 78-79: 102-121.

Robinson, A. 1957. La dimensión óptima de la empresa. Buenos Aires: Ed. El Ateneo.

Salas, V. 1986. ¿Conoce el crecimiento sostenible por su empresa? Alta Dirección, № 127: 83-91.

Suárez, A. 1993. Decisiones óptimas de inversión y financiación en la empresa. Madrid: Pirámide.

Suárez, A 1977. La rentabilidad y el tamaño de las empresas españolas. Económicas y Empresariales, Nㅜ 5: 56-63. 


\section{Revista de}

\section{investigaciones UNAD}

Volumen 13. Número 1. Junio 2014

Suárez, A. 1976. El tamaño y el crecimiento de las empresas españolas. La Ley del efecto proporcional. Económicas y Empresariales, $\mathrm{N}^{\circ}$ 3: 116-132.

Sutton, C. 1983. Economía y estrategias de la empresa. México: Limusa S.A. 